\title{
Editorial
}

Digestion

\section{Computed Tomography Just after Endoscopy as the Preoperative Examination for Safe Percutaneous Endoscopic Gastrostomy}

\author{
Yasushi Adachi Kimishige Akino Hiroaki Mita Takefumi Kikuchi \\ Takao Endo \\ Division of Gastroenterology, Department of Internal Medicine, Sapporo Shirakaba-dai Hospital, \\ Sapporo, Japan
}

Percutaneous endoscopic gastrostomy (PEG) is the most common method of enteral nutrition in patients who require long-term tube feeding and is safer and more cost-effective than surgically placed gastrostomies $[1,2]$. However, there are several cases with difficulty in gastrostomy for morphological reasons [2]. Moreover, PEG could be associated with several potential complications and postoperative death [1-5]. In order to perform PEG more safely and more quickly, we arranged preoperative examination. Abdominal computed tomography (CT) was taken just after endoscopic examination, thus the stomach was filled with air. Then three-dimensional CT (3D-CT) images were constructed to reveal the positional relation between the stomach and surrounding organs, including colon, intestine, liver, spleen, costal margin, and aorta (fig. 1). As these 3D-CT images could be used for simulation, we can insert safely a needle and ultimately the gastrostomy tube into the stomach lumen without damage to adjacent structures (fig. 2). Moreover, 3D-CT could disclose several variances and difficult cases (fig. 2).
We analyzed 160 cases scheduled for PEG and 3D-CT revealed that 36 cases $(22.5 \%)$ have some variances as follows (table 1); deformities of the stomach (13.8\%), abnormal locations of the stomach (3.6\%), and interference in puncture routes by other organs (8.8\%).

The most attractive merit of our method is that we can avoid the suspension of surgery by some preparations, e.g. gas in the transverse colon could be reduced by medication, including probiotics. We can give much attention to both planning and performance of PEG, if 3D-CT simulation showed some difficulties. Moreover, the patients and their families could understand the surgical procedure by the 3D-CT image, especially in cases of some trouble or withdrawal.

This examination is not perfect as re-shrinkage of stomach could be happen due to belching or peristalsis. Although CT fluoroscopy-guided PEG has been reported [6], it is difficult to be performed for all cases. This presurgical examination might bring us much precious information.

\section{KARGER}

E-Mail karger@karger.com

www.karger.com/dig
(C) 2013 S. Karger AG, Basel

$0012-2823 / 13 / 0882-0125 \$ 38.00 / 0$ 

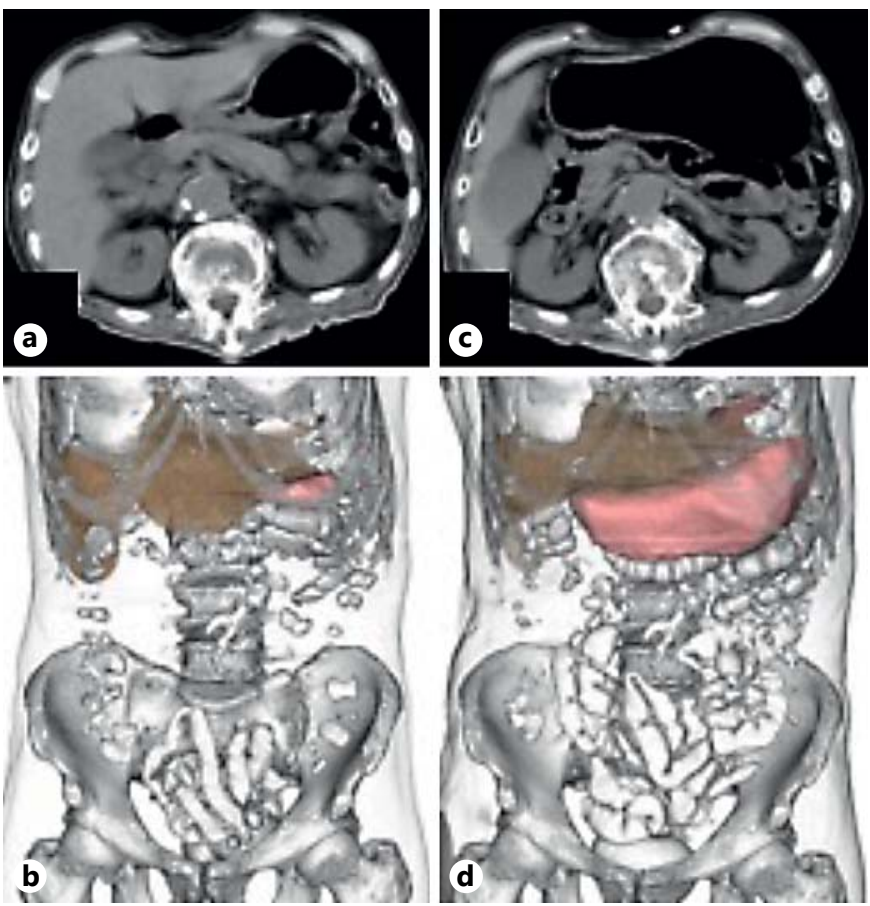

Fig. 1. Difference of 3D-CT images between before and after endoscopic examination. When abdominal CT was examined as usual in hunger, the stomach was shrunk (a). A 3D-CT image showed that the stomach was hidden by adjacent organs (b). CT taken just after endoscopic examination showed that the stomach was filled with air and located by the peritoneal wall (c). The 3D-CT image can illustrate the positional relation between the stomach and surrounding organs as similar to the operation $(\mathbf{d})$.

Table 1. Summary of variance for PEG analyzed by 3D-CT

\begin{tabular}{lcc}
\hline & $\begin{array}{l}\text { Number } \\
\text { of cases }\end{array}$ & $\%$ \\
\hline Normal & 124 & 77.5 \\
Variance & 36 & 22.5 \\
\hline Deformity of the stomach & 22 & 13.8 \\
$\quad$ Steer-horn (hourglass) stomach & 18 & 11.3 \\
$\quad$ Cascade stomach & 1 & 0.6 \\
$\quad$ Resected stomach & 3 & 1.9 \\
Abnormal location of the stomach & 6 & 3.8 \\
$\quad$ Thoracic stomach (herniation) & 6 & 3.8 \\
Interference in routes for & 14 & 8.8 \\
$\quad$ percutaneous needle puncture & 10 & 6.3 \\
$\quad$ Transverse colon & 2 & 1.3 \\
$\quad$ Liver & 2 & 1.3 \\
\hline Ventriculoperitoneal shunt tube & 160 & \\
\hline Total & & \\
\hline
\end{tabular}
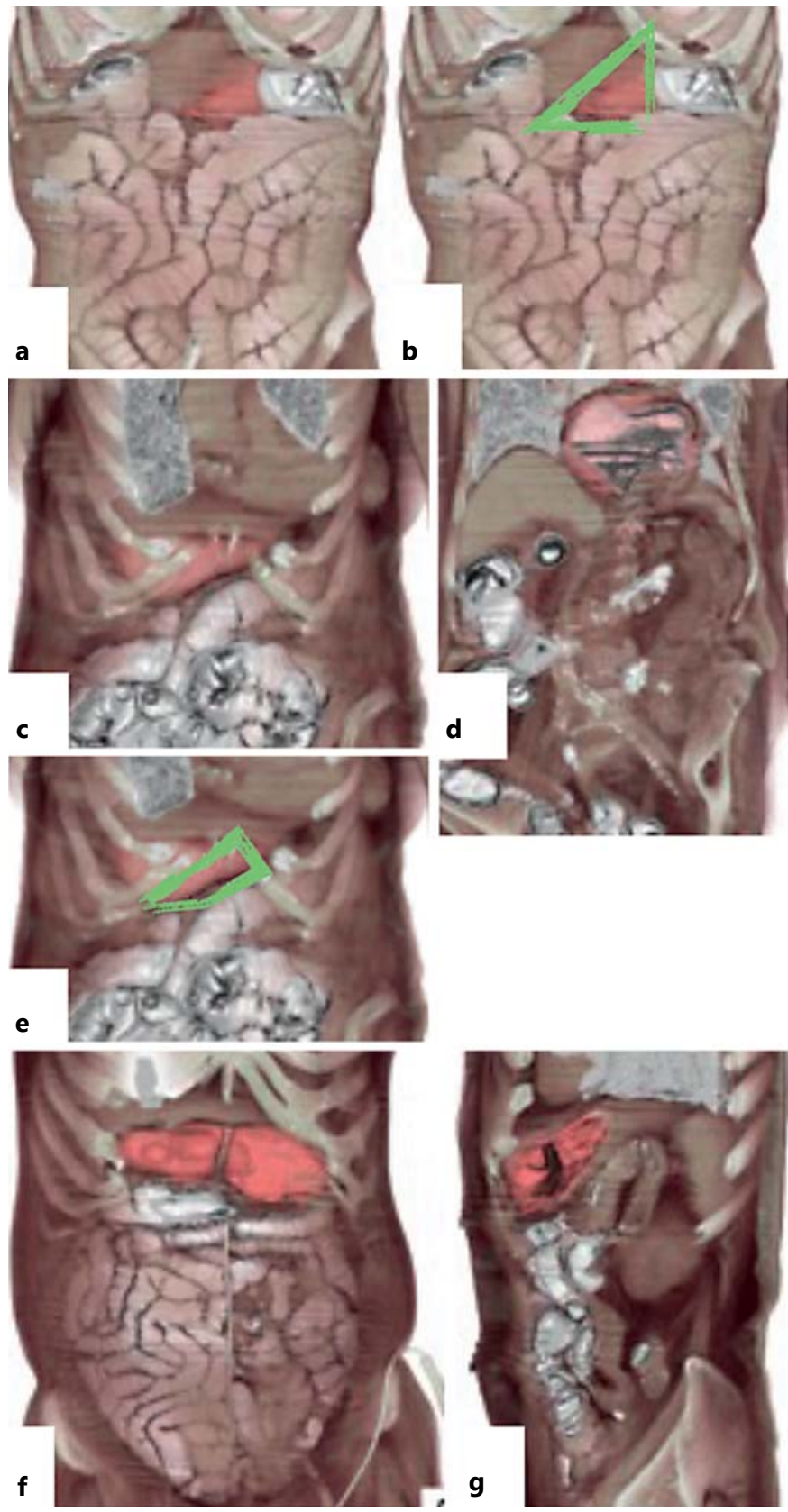

Fig. 2. Benefit of simulations for PEG using 3D-CT, especially in cases with some difficulties. a, b 3D-CT showed a narrow window (green triangle in $\mathbf{b}$ ) for PEG in the patient with a resected stomach. c-e 3D-CT revealed a shallow window (green trapezoid in e) in antrum for insertion of the gastrostomy tube in the patient with a huge sliding hernia. Finally, a jejunal tube was extended from PEG, Jet-PEG [2]. f, g 3D-CT identified a VP shunt tube crossed in the front of a steer-horn stomach, After the stomach was stretched by endoscopy, gastrostomy was performed on the anterior wall of stomach and the tube was not harmed. 


\section{References}

1 Gomes CA Jr, Lustosa SA, Matos D, et al: Percutaneous endoscopic gastrostomy versus nasogastric tube feeding for adults with swallowing disturbances. Cochrane Database Syst Rev 2010;11:CD008096.

$\checkmark 2$ Zopf Y, Rabe C, Bruckmoser T, et al: Percutaneous endoscopic jejunostomy and jejunal extension tube through percutaneous endoscopic gastrostomy: a retrospective analysis of success, complications and outcome. Digestion 2009;79:92-97.
3 Lang A, Bardan E, Chowers Y, et al: Risk factors for mortality in patients undergoing percutaneous endoscopic gastrostomy. Endoscopy 2004;36:522-526.

$\checkmark 4$ Zopf Y, Maiss J, Konturek P, et al: Predictive factors of mortality after PEG insertion: guidance for clinical practice. J Parenter Enteral Nutr 2011;35:50-55.
5 Tominaga N, Shimoda R, Iwakiri R, et al: Low serum albumin level is risk factor for patients with percutaneous endoscopic gastrostomy. Intern Med 2010;49:2283-2288.

$\checkmark 6$ De Bucourt M, Collettini F, Althoff C, et al: CT fluoroscopy-guided percutaneous gastrostomy with loop gastropexy and peel-away sheath trocar technique in 31 amyotrophic lateral sclerosis patients. Acta Radiol 2012;53: 285-291. 